\title{
A!
}

This is an electronic reprint of the original article.

This reprint may differ from the original in pagination and typographic detail.

Slotte, J.; Kilpeläinen, S.; Tuomisto, F.; Räisänen, J.; Nylansted Larsen, A.

\section{Direct observations of the vacancy and its annealing in germanium}

Published in:

Physical Review B

DOI:

10.1103/PhysRevB.83.235212

Published: 01/06/2011

Document Version

Publisher's PDF, also known as Version of record

Please cite the original version:

Slotte, J., Kilpeläinen, S., Tuomisto, F., Räisänen, J., \& Nylansted Larsen, A. (2011). Direct observations of the vacancy and its annealing in germanium. Physical Review B, 83(23), 1-5. [235212].

https://doi.org/10.1103/PhysRevB.83.235212

This material is protected by copyright and other intellectual property rights, and duplication or sale of all or part of any of the repository collections is not permitted, except that material may be duplicated by you for your research use or educational purposes in electronic or print form. You must obtain permission for any other use. Electronic or print copies may not be offered, whether for sale or otherwise to anyone who is not an authorised user. 


\title{
Direct observations of the vacancy and its annealing in germanium
}

\author{
J. Slotte, ${ }^{1}$ S. Kilpeläinen, ${ }^{1}$ F. Tuomisto, ${ }^{1}$ J. Räisänen, ${ }^{2}$ and A. Nylandsted Larsen ${ }^{3}$ \\ ${ }^{1}$ Department of Applied Physics, Aalto University, P.O. Box 11100, FI-00076 AALTO, Finland \\ ${ }^{2}$ Department of Physics, University of Helsinki, P.O. Box 64, FI-00014 Helsinki, Finland \\ ${ }^{3}$ Department of Physics and Astronomy, University of Aarhus, DK 8000 Aarhus C, Denmark
}

(Received 17 January 2011; published 29 June 2011)

\begin{abstract}
Weakly $n$-type doped germanium has been irradiated with protons up to a fluence of $3 \times 10^{14} \mathrm{~cm}^{-2}$ at $35 \mathrm{~K}$ and $100 \mathrm{~K}$ in a unique experimental setup. Positron annihilation measurements show a defect lifetime component of $272 \pm 4$ ps at $35 \mathrm{~K}$ in in situ positron lifetime measurements after irradiation at $100 \mathrm{~K}$. This is identified as the positron lifetime in a germanium monovacancy. Annealing experiments in the temperature interval 35-300 K reveal two annealing stages. The first at $100 \mathrm{~K}$ is tentatively associated with the annealing of the Frenkel pair, the second at $200 \mathrm{~K}$ with the annealing of the monovacancy. Above $200 \mathrm{~K}$ it is observed that mobile neutral monovacancies form divacancies, with a positron lifetime of 315 ps.
\end{abstract}

DOI: 10.1103/PhysRevB.83.235212

PACS number(s): 78.70.Bj, 61.72.Cc, 61.72.Ff, 61.72.jd

\section{INTRODUCTION}

Since silicon has dominated the semiconductor industry for over 50 years, it is also one of the most studied elements. Fundamental properties such as electrical conductivity, heat capacity, etc. have been determined and studied in numerous publications. Furthermore, in the early advent of semiconductor technology it was already realized that point defects, such as impurities, self-interstitials, and vacancies, played an important role in the electrical properties of silicon by introducing energy levels within the forbidden bandgap. Point defects can also contribute to the diffusion properties of dopants and host lattice atoms. Hence, the properties of point defects in silicon are known in great detail, ${ }^{1,2}$ even though silicon can still surprise us. ${ }^{3}$ To a large extent the knowledge of point defects in silicon is owed to the pioneering work by Corbett and Watkins, who succesfully applied the electron paramagnetic resonance (EPR) technique to identify point defects and their annealing temperatures. $2,4,5$

However, when discussing germanium, much is still unknown. Among the fundamental properties that still needs addressing are migration and formation energies of point defects and their annealing temperatures. It has been established that in germanium the vacancy is the dominant diffusion-mediating point defect, both in self-diffusion ${ }^{6-10}$ and impurity diffusion. ${ }^{9}{ }^{11}$ Some experimental work has been done on the determination of the formation and migration energies of the vacancy in germanium. Early work by Whan ${ }^{12}$ estimated the value of the migration energy to be $0.2 \mathrm{eV}$. Ershov et al. ${ }^{13}$ and Shaw ${ }^{14}$ determined the migration energies of the neutral and singly negative vacancy. The values found by Ershov et al. were $0.52 \pm 0.05 \mathrm{eV}$ and $0.42 \pm 0.04 \mathrm{eV}$, respectively. Shaw deduced slightly higher values, $0.7 \mathrm{eV}$ and $1.0 \mathrm{eV}$, respectively. Various authors have performed quenching experiments to determine the formation energy of the singly negatively charged vacancy. ${ }^{6,15-20}$ All of these studies find a formation energy close to $2 \mathrm{eV}$. It should, however, be noted that almost all the estimates above are based on indirect spectroscopic tools. The fact that Ge has several stable isotopes has until now made it impossible to replicate the triumphs of the EPR technique in Si.
For the electronic properties of a semiconductor, the levels in the bandgap of a point defect, i.e. the possible charge states of a defect, are of utmost importance. One of the most common techniques to study defect levels in semiconductors is deeplevel transient spectroscopy (DLTS). Even though germanium has not been as extensively studied as silicon, consensus seems to have been found to some extent regarding the Frenkel pair and the monovacancy. Recently Mesli et al. reported on lowtemperature irradiation-induced point defects in germanium. ${ }^{21}$ This study associated a level at $E_{c}-0.14 \mathrm{eV}$ with the Frenkel pair. This level anneals out at $65 \mathrm{~K}$ in good agreement with previous studies. ${ }^{22,23}$ A level tentatively associated with the $-l=$ transition of the monovacancy is found $0.14 \mathrm{eV}$ above the valence band. ${ }^{21}$ This is in agreement with previous Hall effect measurements which place this level $0.1-0.2 \mathrm{eV}$ above the valence band. ${ }^{19,20}$ Konorova $^{20}$ and Hiraki and Suita ${ }^{19}$ also find a $0 /-$ level close to the valence band at $E_{v}+0.03 \mathrm{eV}$. It can be noted that a perturbed angular correlation spectrocopy study associates an acceptor level of the vacancy at $E_{v}+0.2 \mathrm{eV}$ with the $0 /-$ transition. ${ }^{24}$

The stability of the divacancy at RT has from the point of view of DLTS been a long-time controversy. ${ }^{25}$ Mooney et al. ${ }^{26}$ claim that the divacancy is stable at RT and anneals around $150^{\circ} \mathrm{C}$, whereas more recent studies find no trace of the divacancy in alpha-particle-irradiated germanium..$^{25,27}$ However, in a recent positron annihilation spectroscopy (PAS) study the neutral divacancy was identified and found to be stable at RT. Furthermore, strong evidence of a negative charge state for the divacancy in the bandgap was also reported. ${ }^{28}$ These findings are supported by a recent DLTS study by Petersen et al. ${ }^{29}$

A few low-temperature irradiation PAS studies on germanium have previously been reported. ${ }^{30-32} \mathrm{~A}$ common feature of all these studies is that electron irradiation has been performed at cryogenic temperatures, and the samples have then been transported and measured at liquid nitrogen temperatures. The studies by Moser et al. ${ }^{30}$ and Corbel et al. ${ }^{31}$ show a defect lifetime component $\tau_{2}$ of 290 and 292 ps, respectively. However, no further information is available about the samples, e.g. sample doping, and no direct conclusions are made on the nature of the defect. In the study by Polity and Rudolf ${ }^{32}$ 
a defect-related lifetime of 281 ps is reported, measured at $90 \mathrm{~K}$ and associated with a monovacancy-type defect. However, no decomposition data are presented. A similar lifetime component is observed after annealing at $350 \mathrm{~K}$. It is speculated that at least above RT the 281 ps component could be associated with the $\mathrm{V}_{2} \mathrm{O}$ complex. All of these studies observe an annealing stage at $200 \mathrm{~K}$, which Polity and Rudolf associate with the annealing of the Frenkel pair.

In this paper, we make direct observations of the monovacancy in germanium and its annealing. The measurements are enabled by use of a unique PAS setup. The monovacancies are produced by low-temperature proton irradiation of weakly $n$ type doped (Sb) germanium, with subsequent in situ annealing and PAS lifetime measurements in the temperature interval 35-300 K.

\section{EXPERIMENTAL DETAILS}

The samples, $n$-type germanium ([Sb] $\approx 2 \times 10^{14} \mathrm{~cm}^{-3}$ ), were mounted in the conventional sandwich setup, ${ }^{33}$ with a ${ }^{22} \mathrm{Na}^{+}$-source wrapped in a $2-\mu \mathrm{m}$ aluminum foil, on a copper sample holder in thermal contact with a closed-cycle helium cryostat. For the positron lifetime analysis we used a conventional fast-fast coincidence system with a resolution of $260 \mathrm{ps}$ and a $20 \mu \mathrm{Ci}{ }^{22} \mathrm{NaCl}$ source. The reference (bulk) lifetime in the measurements with this setup was $\tau_{B}=228 \mathrm{ps}$. Prior to the analysis, the positron annihilations in the source material, in the $\mathrm{Al}$ foil covering the source and as positronium, were subtracted from the lifetime spectra. The sample holder had an approximately $80 \mathrm{~mm}^{2}$ aperture for the ion beam. The irradiation was performed with $9-10 \mathrm{MeV}$ protons, with a projected range of approximately $400 \mu \mathrm{m}$ in germanium. For a more detailed description of the unique experimental setup see Ref. 34. To get a homogenous defect distribution within the positron implantation profile, the top sample was ground mechanically to a thickness of approximately $250 \mu \mathrm{m}$. The projected range of the protons in the bottom sample was thus around $150 \mu \mathrm{m}$, which was deep enough to avoid the ion end of range effects in the PAS analysis, as the average positron implantation depth in germanium is $80 \mu \mathrm{m}$. The irradiation was done in steps from a fluence of $1 \times 10^{12} \mathrm{~cm}^{-2}$ up to $3 \times 10^{14} \mathrm{~cm}^{-2}$ at $35 \mathrm{~K}$ in order to be able to monitor the defect production. A fluence of approximately $1 \times 10^{14} \mathrm{~cm}^{-2}$ was needed for a detectable increase in the average positron lifetime, which is a sign of vacancy defects being present in the material. With a typical introduction rate for primary defects in a semiconductor for $10 \mathrm{MeV}$ protons, $\phi=200-800 \mathrm{~cm}^{-1}, 34$ a fluence of $1 \times 10^{14} \mathrm{~cm}^{-2}$ should correspond to a vacancy concentration of the order of $10^{16} \mathrm{~cm}^{-3} \cdot \tau_{\text {ave }}$ also corresponds to the center of mass of the lifetime spectrum and is insensitive to the decomposition process. The statistical error in $\tau_{\text {ave }}$ is of the order of 1 ps. To be able to distinguish between annealing stages some samples were irradiated at $100 \mathrm{~K}$.

The $9-10 \mathrm{MeV}$ proton irradiation caused some activation of the sample. ${ }^{35}$ To avoid an unwanted background in the PAS lifetime spectra from decaying As isotopes, the samples were kept at $35 \mathrm{~K}$ for approximately 4 days prior to the annealing experiments. The annealing was done in $30 \mathrm{~min}$ steps from $35 \mathrm{~K}$, or $100 \mathrm{~K}$, up to RT, with measurements at $35 \mathrm{~K}$ in between annealing steps.

\section{RESULTS}

Figure 1 shows the average positron lifetime [Fig. 1(c)] after irradiation at $35 \mathrm{~K}$ together with the decomposed defect lifetime component $\tau_{2}$ [Fig. 1(a)] and $\tau_{1}$ [Fig. 1(b)]. Directly after irradiation the average lifetime $\tau_{\text {ave }}$ is approximately 245 ps. Two distinct annealing steps can be observed in the average lifetime; a reduction in average lifetime by approximately $6 \mathrm{ps}$ in the temperature interval $110-160 \mathrm{~K}$ and a subsequent similar increase in the interval 200-230 K.

From the decomposition of the lifetime spectra it was evident that the standard trapping model with two positron states was not adequate enough to describe the spectra. $\tau_{1}$ was found to be clearly above the bulk lifetime of $228 \mathrm{ps}$ in the annealing interval $70-110 \mathrm{~K}$, indicating that a second defect lifetime is mixed into $\tau_{1}$. This effect seems to disappear after the first annealing step (above $200 \mathrm{~K}$ ), after which $\tau_{1}$ behaves according to the two-state model. Hence, the authors emphasize that these points below $200 \mathrm{~K}$ for $\tau_{1}$ and $\tau_{2}$ in



FIG. 1. (Color online) (a) The higher lifetime component $\tau_{2}$, (b) the lower lifetime component $\tau_{1}$, and (c) the average positron lifetime $\tau_{\text {ave }}$. The solid curve has been drawn to guide the eye. The inset shows $\tau_{2}, \tau_{1}$, and $\tau_{\text {ave }}$ after RT annealing and measured at RT. The open gray symbols for $\tau_{1}$ and $\tau_{2}$ cannot be physically interpreted since the two state trapping model obviously fails below $200 \mathrm{~K}$. 


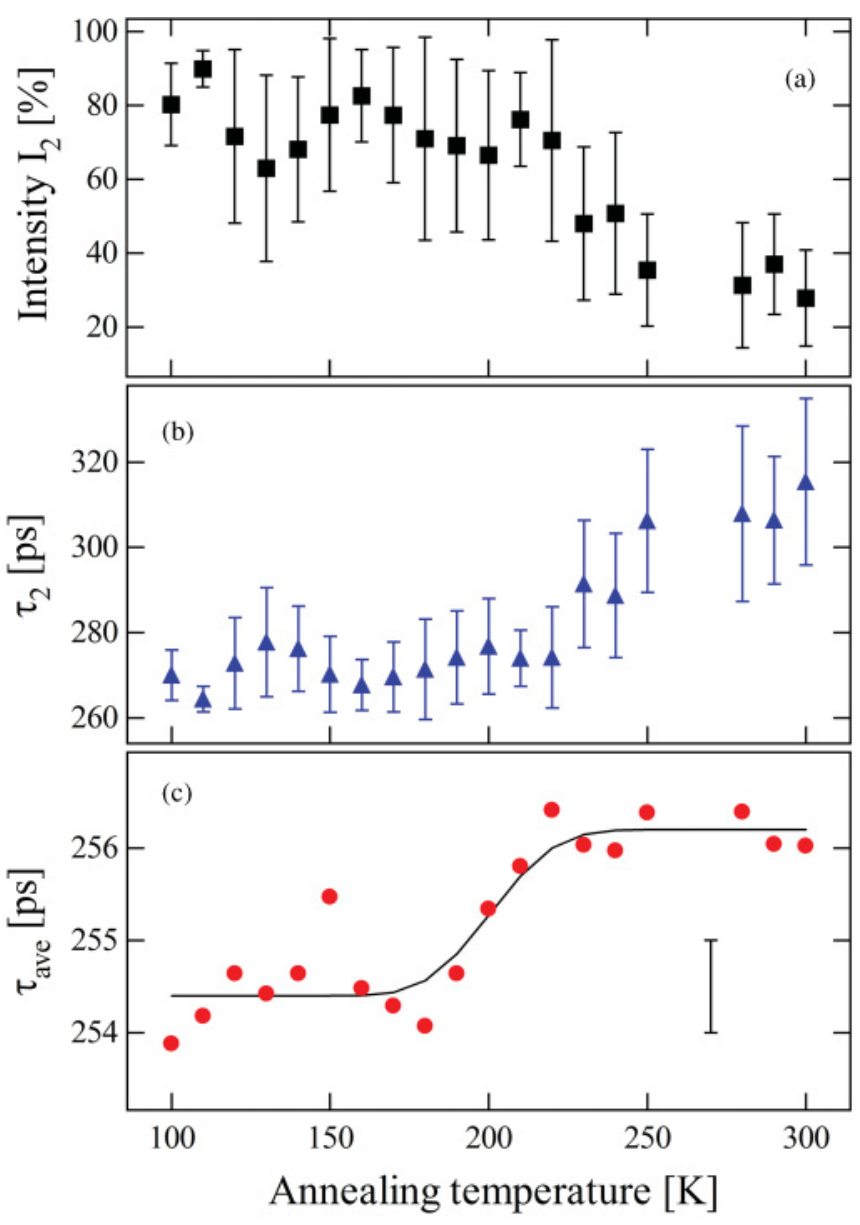

FIG. 2. (Color online) (a) The intensity of the higher lifetime component $I_{2}$, (b) the higher lifetime component $\tau_{2}$, and (c) the average positron lifetime $\tau_{\text {ave }}$. The solid curve has been drawn to guide the eye.

Fig. 1 from the failed decomposition cannot be physically interpreted.

As the results in Fig. 1 clearly indicate that vacancy-type defects are still evolving at RT, we monitored this progress after the sample was removed from the cryostat and kept in air. After 14 days $\tau_{\text {ave }}$ had increased to $252 \mathrm{ps}$, and after 28 days $\tau_{\text {ave }}$ had stabilized at 268 ps. Once the average lifetime had stabilized, successful decomposition of the spectrum was possible and a defect lifetime $\tau_{2}$ of 315 ps was obtained; see inset in Fig. 1.

To avoid problems with the decomposition of the spectra and to be able to unambiguously identify the trapping defects, we performed a second irradiation at $100 \mathrm{~K}$. The results from the annealing experiment can be seen in Fig. 2 with the measurement temperature being again $35 \mathrm{~K}$. As expected, the annealing stage around $100 \mathrm{~K}$ was now absent, and a successful two-component decomposition was possible. A value of $272 \pm 4$ ps was obtained for the decomposed defect lifetime $\tau_{2} . \tau_{\text {ave }}$ stayed approximately constant at a value of $254 \mathrm{ps}$ up to an annealing temperature of $200 \mathrm{~K}$, where an increase in the average lifetime to $256 \mathrm{ps}$ was observed. This increase was accompanied by a continuous increase in $\tau_{2}$.

\section{DISCUSSION}

Previous studies ${ }^{21,22}$ have associated an annealing stage around $70 \mathrm{~K}$ with the Frenkel pair in Ge. This annealing temperature coincides fairly well with the first annealing stage observed in Fig. 1(c). Hence, we tentatively conclude that the first annealing stage observed at $100 \mathrm{~K}$ is due to annealing of the Frenkel pair. It should be noted that the concentration range in our measurements is several orders of magnitude higher than in, e.g. the study by Mesli et al. ${ }^{21}$ Therefore the observation of the annealing kinetics will be slower compared with DLTS experiments, and several annealing steps are hence needed for the Frenkel pair to anneal in the PAS spectra. It should also be noted that PAS is only sensitive to the vacancy and not to the Frenkel pair as a whole. A possibility is therefore that the annealing stage at $100 \mathrm{~K}$ is a recombination of an isolated monovacancy and an interstitial, with the interstitial being the mobile defect.

Even though the behavior of the first lifetime component is in accordance with the two-state model of PAS above an annealing temperature of approximately $120 \mathrm{~K}$, no identification of the primary positron trap left in the sample is possible. As can be seen from Fig. 1(a) the defect component $\tau_{2}$ is increasing continuously above $200 \mathrm{~K}$, as is the average positron lifetime. This is a clear indication that the open volume of the vacancy defects that still remain in the sample is increasing.

As noted, the annealing stage above $100 \mathrm{~K}$ was not observed after irradiation at $100 \mathrm{~K}$. This is in accordance with the interpretation that this stage was due to the annealing of the Frenkel pair, which should not be stable when the irradiation temperature is above approximately $80 \mathrm{~K}$. The identified defect lifetime component measured at $35 \mathrm{~K}$ after irradiation at $100 \mathrm{~K}$ is 272 ps. This is clearly a lifetime related to a defect of monovacancy size. ${ }^{33,36}$ The concentration of these vacancy defects is $1.3 \times 10^{17} \mathrm{~cm}^{-3}$, calculated from the conventional trapping model. ${ }^{33}$ This gives a defect introduction rate of approximately $300 \mathrm{~cm}^{-1}$ in agreement with the previously mentioned estimates. Since no impurities are present in a high-enough concentration the positron trap related to the 272 ps lifetime cannot be a complex of a monovacancy and an impurity. Thus, this lifetime is identified as the positron lifetime in a pure monovacancy. The intensity of this lifetime component stays constant [Fig. 2(a)] until the monovacancy starts to anneal at approximately $200 \mathrm{~K}$.

DLTS studies on low-temperature irradiated Ge assign a double acceptor level at $E_{V}+0.12 \mathrm{eV}$ to the monovacancy, ${ }^{21}$ whereas Haesslein et al., ${ }^{24}$ using perturbed angular correlation spectroscopy, assign a single acceptor state for the vacancy at $E_{V}+0.2 \mathrm{eV}$. However, later investigations of the data in Ref. 21 show that the level observed is most likely the single acceptor level, ${ }^{37}$ in agreement with Haesslein et al. Nevertheless, in the case of this study, which is performed on weakly $n$-type material, the monovacancy should at a first glance be negatively charged. However, the vacancy concentration exceeds the charge carrier concentration by two orders of magnitude, i.e. there are only charge carriers to ionize a small fraction of the monovacancies, and the majority of the monovacancies should thus be neutral.

As can be seen from Fig. 2 the monovacancy starts to anneal at approximately $200 \mathrm{~K}$ and, similarly to the results in 


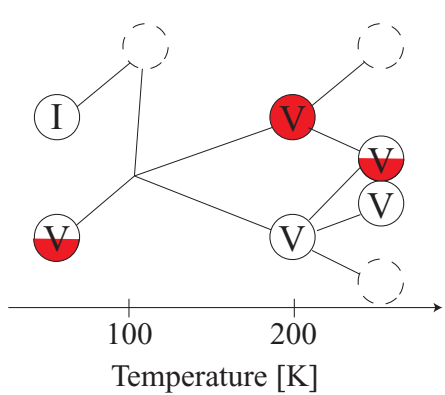

FIG. 3. (Color online) Schematics of the vacancy reactions in the temperature range $35-300 \mathrm{~K}$. The coloring on the vacancies indicates charge state, red negative or transparent neutral, a dashed circle indicates annealing.

Fig. 1, the average lifetime and the defect lifetime component $\tau_{2}$ increase. The increase in the defect lifetime clearly indicates that the average open volume of the positron trapping defect is increasing. As $\tau_{2}$ stabilizes to $315 \mathrm{ps}$ in the RT annealing of the sample in Fig. 1 and the $300 \mathrm{~K}$ annealing in Fig. 2 , we conclude that at least a considerable fraction of the monovacancies form divacancies when they become mobile at temperatures above $200 \mathrm{~K}$. The calculated concentration of divacancies after the $300 \mathrm{~K}$ annealing in Fig. 2 is approximately $4 \times 10^{16} \mathrm{~cm}^{-3}$, whereas the vacancy concentration after irradiation is approximately $1.3 \times 10^{17} \mathrm{~cm}^{-3}$, i.e. roughly $60 \%$ of the vacancies form divacancies.

The defect lifetime for the divacancy is slightly lower compared with the 330-ps lifetime which was identified as the positron lifetime in a neutral divacancy in Ref. 28. As the average lifetime at RT in the sample used in this study is considerably lower as compared with that of Ref. 28, i.e. the concentration of divacancies is considerably lower, it can be speculated if the 315 ps lifetime is the positron lifetime in a negatively charged divacancy. That could be the case if there are enough charge carriers to ionize at least a considerable fraction of the divacancies.

In the schematics of Fig. 3, we summarize the vacancy reactions deduced from the measurements in this paper. In the first annealing stage around $100 \mathrm{~K}$ a considerable fraction of vacancies are annealed when the close Frenkel pair or an isolated monovacancy and interstital recombine. The vacancies left in the samples after this annealing stage become mobile at approximately $200 \mathrm{~K}$ and either anneal or pair with another vacancy to form more stable divacancies.

\section{CONCLUSIONS}

We have used an experimental PAS setup to directly observe the monovacancy in germanium and to study the annealing stages of this defect. In situ positron annihilation spectroscopy measurements on low-temperature proton irradiated weakly $n$-type doped germanium show two annealing stages; the first stage at $100 \mathrm{~K}$ was associated with the annealing of the close Frenkel pair and the second stage at $200 \mathrm{~K}$ with the annealing of the monovacancy. A positron lifetime of 272 ps was identified as the positron lifetime in the germanium monovacancy. In the annealing experiments performed at temperatures above $200 \mathrm{~K}$ it was observed that neutral monovacancies paired to form divacancies.
${ }^{1}$ J. Vanhellemont and E. Simoen, J. Electrochem. Soc. 154, H572 (2007).

${ }^{2}$ G. D. Watkins, in Deep Centers in Semiconductors, edited by T. Pantelides (Gordon Breach, New York, 1986).

${ }^{3}$ A. N. Larsen, A. Mesli, K. B. Nielsen, H. K. Nielsen, L. Dobaczewski, J. Adey, R. Jones, D. W. Palmer, P. R. Briddon, and S. Oberg, Phys. Rev. Lett. 97, 106402 (2006).

${ }^{4}$ G. D. Watkins and J. W. Corbett, Phys. Rev. 134, A1359 (1964).

${ }^{5}$ G. D. Watkins, IEEE Trans. Nucl. Sci. NS-16, 13 (1969).

${ }^{6}$ A. Giese, N. A. Stolwijk, and H. Bracht, Appl. Phys. Lett. 77, 642 (2000).

${ }^{7}$ M. Werner, H. Mehrer, and H. D. Hochheimer, Phys. Rev. B 32, 3930 (1985).

${ }^{8}$ H. D. Fuchs, W. Walukiewicz, E. E. Haller, W. Dondl, R. Schorer, G. Abstreiter, A. I. Rudnev, A. V. Tikhomirov, and V. I. Ozhogin, Phys. Rev. B 51, 16817 (1995).

${ }^{9}$ M. Naganawa, Y. Shimizu, M. Uematsu, K. M. Itoh, K. Sawano, Y. Shiraki, and E. E. Haller, Appl. Phys. Lett. 93, 191905 (2008).

${ }^{10}$ E. Hüger, U. Tietze, D. Lott, H. Bracht, D. Bougeard, E. E. Haller, and H. Schmidt, Appl. Phys. Lett. 93, 162104 (2008).

${ }^{11}$ S. Brotzmann, H. Bracht, J. L. Hansen, A. N. Larsen, E. Simoen, E. E. Haller, J. S. Christensen, and P. Werner, Phys. Rev. B 77, 235207 (2008).

${ }^{12}$ R. E. Whan, Phys. Rev. 140, 690 (1965).
${ }^{13}$ S. N. Ershov, V. A. Pantaleev, S. N. Nagornkh, and V. V. Chernyakhovskii, Sov. Phys. Solid State 19, 187 (1977).

${ }^{14}$ D. Shaw, Phys. Status Solidi B 72, 11 (1975).

${ }^{15}$ S. Mayburg, Phys. Rev. 95, 38 (1954).

${ }^{16}$ R. A. Logan, Phys. Rev. 101, 1455 (1956).

${ }^{17}$ A. G. Tweet, Phys. Rev. 106, 221 (1957).

${ }^{18}$ A. G. Tweet, J. Appl. Phys. 30, 2002 (1959).

${ }^{19}$ A. Hiraki and Y. Suita, Technol. Rep. Osaka Univ. 15, 65 (1965).

${ }^{20}$ L. F. Konorova, Sov. Phys. Solid State 10, 2233 (1969).

${ }^{21}$ A. Mesli, L. Dobaczewski, K. B. Nielsen, V. Kolkovsky, M. C. Petersen, and A. N. Larsen, Phys. Rev. B 78, 165202 (2008).

${ }^{22}$ V. Emtsev, Mater. Sci. Semicond. Process. 9, 580 (2006).

${ }^{23}$ T. A. Callcott and J. W. Mackay, Phys. Rev. 161, 698 (1967).

${ }^{24}$ H. Haesslein, R. Sielemann, and C. Zistl, Phys. Rev. Lett. 80, 2626 (1998).

${ }^{25}$ V. Kolkovsky, M. C. Petersen, A. N. Larsen, and A. Mesli, Mater. Sci. Semicond. Process. 11, 336 (2008).

${ }^{26}$ P. M. Mooney, F. Poulin, and J. C. Bourgoin, Phys. Rev. B 28, 3372 (1983).

${ }^{27}$ V. Kolkovsky, M. C. Petersen, and A. N. Larsen, Appl. Phys. Lett. 90, 112110 (2007). 
${ }^{28}$ K. Kuitunen, F. Tuomisto, J. Slotte, and I. Capan, Phys. Rev. B 78, 033202 (2008).

${ }^{29}$ M. C. Petersen, A. N. Larsen, and A. Mesli, Phys. Rev. B 82, 075203 (2010).

${ }^{30}$ P. Moser, J. L. Pautrat, C. Corbel, and P. Hautojärvi, in Positron Annihilation, edited by P. C. Jain, M. Singru, and K. P. Gopinathan (World Scientific, Singapore, 1985).

${ }^{31}$ C. Corbel, P. Moser, and M. Stucky, Ann. Chim. (Paris) 733 (1985).

${ }^{32}$ A. Polity and F. Rudolf, Phys. Rev. B 59, 10025 (1999).
${ }^{33}$ K. Saarinen, P. Hautojärvi, and C. Corbel, in Identification of Defects in Semiconductors, edited by M. Stavola (Academic, New York, 1998).

${ }^{34}$ S. Väyrynen, P. Pusa, P. Sane, P. Tikkanen, J. Räisänen, K. Kuitunen, F. Tuomisto, J. Härkönen, I. Kassamakov, E. Tuominen et al., Nucl. Instrum. Methods A 572, 978 (2007).

${ }^{35}$ K. Krivan and V. Krivan, Anal. Chem. 295, 348 (1979).

${ }^{36}$ R. Krause-Rehberg and H. S. Leipner, Positron Annihilation in Semiconductors (Springer, Berlin, 1999).

${ }^{37}$ M. C. Petersen, Ph.D. thesis, Department of Physics and Astronomy, University of Aarhus, 2010. 